\title{
Surface convection and red-giant radius measurements
}

\author{
L. Piau ${ }^{1}$, P. Kervella ${ }^{2}$, S. Dib ${ }^{3}$, and P. Hauschildt ${ }^{4}$ \\ 1 Service d'astrophysique, CEA Saclay, 91191 Gif-sur-Yvette, France \\ e-mail: laurent.piau@cea.fr \\ 2 LESIA, Observatoire de Paris, CNRS UMR 8109, UPMC, Université Paris Diderot, 5 place Jules Janssen, 92195 Meudon, France \\ e-mail: Pierre.Kervella@obspm.fr \\ 3 Astrophysics Group, Imperial College of Science, Technology and Medicine, London SW7 2AZ, UK \\ ${ }^{4}$ Hamburger Sternwarte, Gojenbergsweg 112, 21029 Hamburg, Germany
}

Received 16 March 2010 / Accepted 10 October 2010

\begin{abstract}
Phenomenological models of convection adopt characteristic length scales that are chosen to fit solar or stellar observations. We investigate whether changes in these length scales are required between the Sun and low mass stars on the red giant branch (RGB). The question is addressed jointly in the frameworks of the mixing length theory and the full spectrum of turbulence model. For both models, the convective length scale is assumed to be a fixed fraction of the local pressure scale height. We use constraints provided by the observed effective temperatures and linear radii independently. We consider a sample of 38 nearby giants and subgiants for which surface temperatures and luminosities are known accurately and the radii are determined by interferometry to better than $10 \%$. We computed dedicated models for the few cases where the stellar masses were determined by asteroseismological measurements. First we calibrated the solar models. With the same physics, we then computed RGB models for masses between $0.9 M_{\odot}$ and $2.5 M_{\odot}$ and metallicities ranging from $[\mathrm{Fe} / \mathrm{H}]=-0.34$ to solar. The evolution is followed up to $10^{3} L_{\odot}$. Special attention is given to the opacities and the non-grey atmosphere models used as boundary conditions for which the model of convection is the same as in the interior. For both the mixing length theory and the full spectrum of turbulence models, the characteristic solar length scale for convection has to be slightly reduced to fit the lower edge of the observed RGB. The corresponding models also agree more closely with the expected mass distribution on the RGB and the seismic constraints. These results are robust regardless of effective temperatures determined spectroscopically or radii determined interferometrically are used.
\end{abstract}

Key words. stars: low-mass - convection - stars: evolution - asteroseismology

\section{Introduction}

The modelling of solar and stellar surface convection requires hydrodynamical radiative transfer computations (Nordlund et al. 2009). These tridimensional calculations are extremely timeconsuming because convection zone motions are characterized by short timescales and huge turbulence. Therefore, in spite of currently available computational power, the direct modelling of convection does not rely on first principles only but still requires simplifying assumptions.

Direct observations can constrain the properties of outer convection in stars. The Sun's radius is routinely used to calibrate the main free parameter of the mixing length theory, $\alpha_{\mathrm{mlt}}$, the characteristic length scale of convection in this model being generaly $\alpha_{\mathrm{mlt}} H_{\mathrm{p}}$ where $H_{\mathrm{p}}$ denotes the local pressure scale height. It is customary to assume that the solar $\alpha_{\text {mlt }}$ applies throughout stellar evolution and some recent studies have suggested this to be a valid hypothesis for low-mass red giant stars (Ferraro et al. 2006; VandenBerg et al. 2008). However, hydrodynamical convection calculations (Ludwig et al. 1999) show that slightly different $\alpha_{\text {mlt }}$ should be used depending on the effective temperature (hereafter $T_{\text {eff }}$ ) and the surface gravity (hereafter $\log g$ ). Furthermore, the solar $\alpha_{\text {mlt }}$ appears inappropriate to describe outer convection in red giants in the 3-20 $M_{\odot}$ mass range (Stothers \& Chin 1997) and during the final stages of stellar evolution (D'Antona \& Mazzitelli 1996). Thus the nearby Galactic red giants provide interesting insights into the mixing length theory (hereafter MLT). Besides MLT, another local treatment of convection is increasingly used: the full spectrum of turbulence model (hereafter FST; Canuto et al. 1996). This approach is more physically consistent than the MLT and shows closer agreement with numerous sets of observations (Mazzitelli 1999, and references therein, Samadi et al. 2006).

As red giants and solar surface conditions $\left(T_{\text {eff }}, \log g\right)$ strongly differ, they offer an adequate opportunity to check the MLT and the FST empirically. Such analyses have been made in recent years. However, in the case of the nearby red giants, the usual observational constraints, absolute luminosity and $T_{\text {eff }}$ are now supplemented by direct interferometric radii measurements and the new asterosesimic constraints. In this work, we aim to use these new data to estimate $\alpha_{\text {mlt }}$ and $\alpha_{\text {cgm }}$ and their possible variation from the Sun to Galactic red giants. The analysis is performed for both convection models using constraints on effective temperatures and radii independently. In Sect. 2, we present the physical inputs to our stellar evolution code. We describe in detail the treatment of convection and the atmosphere modelling. In Sect. 3, we present and discuss the observation sample. The calibration of the convective length scales based on the Sun is described in Sect. 4. Section 5 examines the MLT and the FST model of convection constrained by the red giant branch stars. We discuss our results and conclusions in Sect. 6.

\section{The evolution code and the models}

We use the CESAM code (Morel 1997), a hydrostatic onedimensional stellar evolution code ${ }^{1}$. The standard version of

${ }^{1}$ A list of the scientific publications using CESAM is available at http://www.oca.eu/morel/articles.html 
CESAM is however significantly modified here: to allow the computation of the stellar structure during the late stages of evolution, we altered the usual integration variables of CESAM (see Appendix A). The evolution is computed from the zero-age main sequence up to a luminosity of $10^{3} L_{\odot}$ on the red giant branch (hereafter RGB) for stars less massive than $2 M_{\odot}$. For more massive stars, the models are evolved to the end of helium core burning on the early asymptotic giant branch (hereafter eAGB). The nuclear reaction rates are based on the NACRE compilation (Angulo et al. 1999). The network includes the proton-proton chains, the $\mathrm{CNO}$ cycle, and $3 \alpha$ and ${ }^{12} \mathrm{C}(\alpha, \gamma){ }^{16} \mathrm{O}$ reactions. The neutrino losses are computed according to the analytical fits of Itoh et al. (1996). Microscopic diffusion is taken into account in the solar calibration models (Sect. 4) and more generally for all the models down to an effective temperature of $5000 \mathrm{~K}$. This temperature roughly corresponds to the maximum extent in mass of the outer convection zone near the base of the RGB, the socalled first dredge-up. Owing to the deep convective mixing, the subsequent evolution of diffusive and non-diffusive models become indistinguishable ${ }^{2}$ (see Salaris et al. 2002 and references herein) and diffusion is not taken into account below $T_{\text {eff }}=5000 \mathrm{~K}$.

The calculation of solar radius or RGB/eAGB radii models critically depends on the physical assumptions and inputs used in the outer layers. There are four, which we summarize below:

i) The opacities: the low temperature opacities dominated by metallicity effects are critical in determining the effective temperature of giant stars at a given luminosity (Salaris et al. 2002). Below $\log T=3.75$, we use the data from Ferguson et al. $(2005)^{3}$. In the higher temperature regime, we use the OPAL opacities computed from the Lawrence Livermore National Laboratory web interface ${ }^{4}$. Both opacity sets correspond to the solar metal repartition advocated by Asplund et al. (2005). The electron conductive opacities that are only significant in the degenerate helium cores of red giants follow the Itoh et al. (1983) prescription. We model stars well below the RGB tip and using the more recent prescription of conductive opacities by Cassisi et al. (2007) would likely leave the evolution tracks unchanged as noticed by these authors.

ii) The convection: two simplified local treatments of convection are investigated: the mixing length theory and the full spectrum of turbulence model. We chose a prescription of the MLT very similar to that of Böhm-Vitense (1958), whose exact description is given in the appendix of Piau et al. (2005). The formulation of the FST is that of Canuto et al. (1996) and referred as CGM hereafter. The detailed equations are provided in the second appendix to this article. For the CGM approach, we adopt a characteristic length scale $\Lambda=\alpha_{\text {cgm }} H_{\mathrm{p}}$ in the equations - where $H_{\mathrm{p}}$ is the pressure scale height -. The CGM version we use is a simplified version rather than the true version of the CGM, where $\Lambda$ is the distance to the convection/radiation regime boundary. Finally we have two length-scale parameters for the two treatments of convection: $\alpha_{\mathrm{mlt}}$ and $\alpha_{\mathrm{cgm}}$. In the occurrence of convective cores, i.e. for

\footnotetext{
${ }^{2}$ However, we recall that even if the diffusive and non-diffusive models reach the same RGB they do not do so at the same age because the main sequence downward diffusion of helium and heavy elements accelerates the evolution at that stage.

${ }^{3}$ Publicly available at

http://webs.wichita.edu/physics/opacity/

${ }^{4}$ http://physci.1lnl.gov/Research/OPAL/new.html
}

stars more massive than $1.2 M_{\odot}$, we assume convective overshooting ranging from $0.1 H_{\mathrm{p}}$ to $0.2 H_{\mathrm{p}}$ (Claret 2007).

iii) The atmosphere modelling: we define the atmosphere as the region where the Rosseland optical depth is less than $\tau_{b}=$ 20. The outer boundary conditions to the internal structure are taken at this depth where the diffusion equation is valid (Morel et al. 1994). In late-type stars, the convection straddles this atmosphere/interior boundary and because the MLT and the CGM model predict different temperature gradients, it is important to use the same treatment of convection above and below this limit (Montalban et al. 2001; Montalban et al. 2004). In addition, molecular lines have a significant impact on atmosphere structures when $T_{\text {eff }}<5000 \mathrm{~K}$. For these reasons, we introduce two series of non-grey atmosphere models as outer boundary conditions. The first series of temperature-optical depth relations $\left(T(\tau)^{4}=T_{\text {eff }}^{4} f_{\text {grid }}(\tau)\right)$ is computed with the PHOENIX/1D atmosphere code where the convection is handled using the MLT. The second series of temperature-optical depth relations is computed with the ATLAS12 atmosphere code (Castelli 2005). We modified ATLAS12 to use the CGM version of the FST model of convection instead of the MLT. The change is directly inspired by the implementation of the FST to the Atlas9 code by Kupka (1996). The characteristic length scales adopted for the convection in both atmospheric sets is $0.5 H_{\mathrm{p}}$ as suggested by the solar and the cool dwarfs Balmer lines (Samadi et al. 2006; Gardiner et al. 1999). The use of the same convection model in the atmosphere and interior is necessary for consistency. In the proper treatment of the CGM, the convection length scale $\Lambda$ is the distance to the convection/radiation regime boundary. This mimics the increase in the convective efficiency with depth (Heiter et al. 2002) and the associated smooth change in the thermal gradient. However, this treatment is not handled by our code where $\Lambda=\alpha_{\text {cgm }} H_{\mathrm{p}}$. In this framework, it is impossible to fit the solar radius when using in the interior the (small) atmosphere $\alpha_{\mathrm{cgm}}$ required by the Balmer lines. The sudden change in $\alpha_{\mathrm{cgm}}$ (or $\alpha_{\mathrm{mlt}}$ ) from the atmosphere to the interior should induce a discontinuity in the thermal gradient at the limit between the interior and the atmosphere. To smooth this out, we perform a linear interpolation with optical depth on the temperature gradients: provided $\nabla_{\mathrm{a}}$ and $\nabla_{\mathrm{i}}$ are the temperature gradients $\left(\nabla=\frac{\operatorname{dn} T}{\operatorname{dn} P}\right)$ computed using, respectively, the atmosphere and interior formalisms, we compute the gradient $\nabla$ at the the optical depth $\tau<\tau_{\mathrm{b}}$ following:

$\nabla=(1-x(\tau)) \nabla_{a}+x(\tau) \nabla_{i}$

where $x(\tau)=\max \left(0,(\tau-1) /\left(\tau_{b}-1\right)\right)$. Thus, the atmospheric gradient is considered where $\tau<1$, the interior gradient where $\tau>\tau_{\mathrm{b}}$, and a linear combination of them where $1<\tau<\tau_{\mathrm{b}}$.

Both atmosphere grids were computed with the solar composition of Asplund et al. (2005). In both of them, there is a model every $100 \mathrm{~K}$ on the $[4000,6400] \mathrm{K}$ effective temperature range and every 0.5 on the $[0.5,5.5]$ range of decimal $\log$ arithm of surface gravity $(\log g)$. For every stellar model, we perform a linear interpolation in $T_{\text {eff }}$ and $\log g$ using the four closest neighbours on the grid to get the $f(\tau)$ theoretical relations. Together with $T_{\text {eff }}$, this relation provides the temperature atmosphere profile $T(\tau)^{4}=T_{\text {eff }}^{4} f(\tau)$. For the most massive stars modelled, $T_{\text {eff }}>6400 \mathrm{~K}$ on the main sequence and the $f_{\text {grid }}(\tau)$ function is not available. In this case, we use the $f_{\text {grid }}(\tau)$ at $6400 \mathrm{~K}$ and the closest $\log g$. 
iv) The equation of state (hereafter EOS): we use the OPAL 2005 EOS tables for Population I stars. Although the effect of the EOS is somewhat less important than the effects of opacities or atmosphere boundary conditions (Salaris et al. 2002), it still determines the temperature gradient in the convection zone and therefore affects the radius.

We make a final comment on the composition of the models before moving to the next section. The atmosphere $T(\tau)$ relations are computed especially for the Asplund et al. (2005) composition and metal repartition. The EOS and opacities in both low and high temperature regimes assume the same metal repartition. However, we do not only consider the solar composition. The spectroscopic analyses of the red giants of our sample suggest that they to have on average a slightly lower metallicity than the Sun and small but non-negligible composition differences (Sect. 5.2). The total metal mass fraction $Z$ and helium mass fraction $Y$ are changed to explore the impact of composition.

\section{The observations}

The selection of our sample of stars was performed in two steps. We first queried the CHARM2 catalogue (Richichi et al. 2005) to obtain all direct measurements of giant and subgiant angular diameters up to 2004, with effective temperatures in the range from $5000 \mathrm{~K}$ to $5500 \mathrm{~K}$. The giants from the Mark III survey of Hutter et al.'s (1989) were removed from the sample, as most of these measurements present strong biases due to calibration uncertainties (see their Sect. IV, for details). We then searched the literature for more recent observations, and added the measurement of $\gamma$ Sge by Wittkowski et al. (2006), $\delta$ Eri and $\xi$ Hya by Thévenin et al. (2005), and the recent high accuracy CHARA/FLUOR measurements of $\epsilon$ Oph (Mazumdar et al. 2009) and $\eta$ Ser (Mérand et al. 2010). When several independent angular diameter measurements were available for the same star, we combined them into a single value taking into account their original error bars and the consistency of the different measurements. The conversion of uniform disk angular diameters to limb-darkened values was done using linear limbdarkening coefficients by Claret (1995), which are based on stellar atmosphere models by Kurucz (Kurucz 1993). We are aware that the plane parallel ATLAS9 limb-darkening coefficients by Claret (1995) are not optimal for cool, giant stars with low effective gravities, but their differences from those of the PHOENIX models are very small for the selected sample. This difference amounts typically to a few per mille of the stellar size. Choosing the Claret (1995) values has the advantage of preserving the internal consistency of the sample, as the ATLAS9 models are the standard for the quoted interferometric measurements. A comparison of the measured angular diameters with predictions from the $F_{K}(V-K)$ surface brightness-color relations calibrated by Kervella et al. (2004a) on Cepheid supergiants is presented in Fig. 1. The agreement is satisfactory within the uncertainties, with no systematic bias at a level of $2 \%$. We note that these relations, established for supergiants, are almost identical to the relations calibrated by Kervella et al. (2004b) using dwarf and subgiants. This is an indication that the infrared surface brightness-color relations are universal for all classes of FK stars.

The spectroscopic effective temperature $T_{\text {eff }}$, effective gravity $\log g$, and metallicity $[\mathrm{Fe} / \mathrm{H}]$ of each star in our sample were taken from Cayrel de Strobel et al.'s (2001) catalogue, except for $\gamma$ Sge and $\xi$ Hya for which we used the $T_{\text {eff }}$ values determined in the articles reporting their interferometric angular diameters.

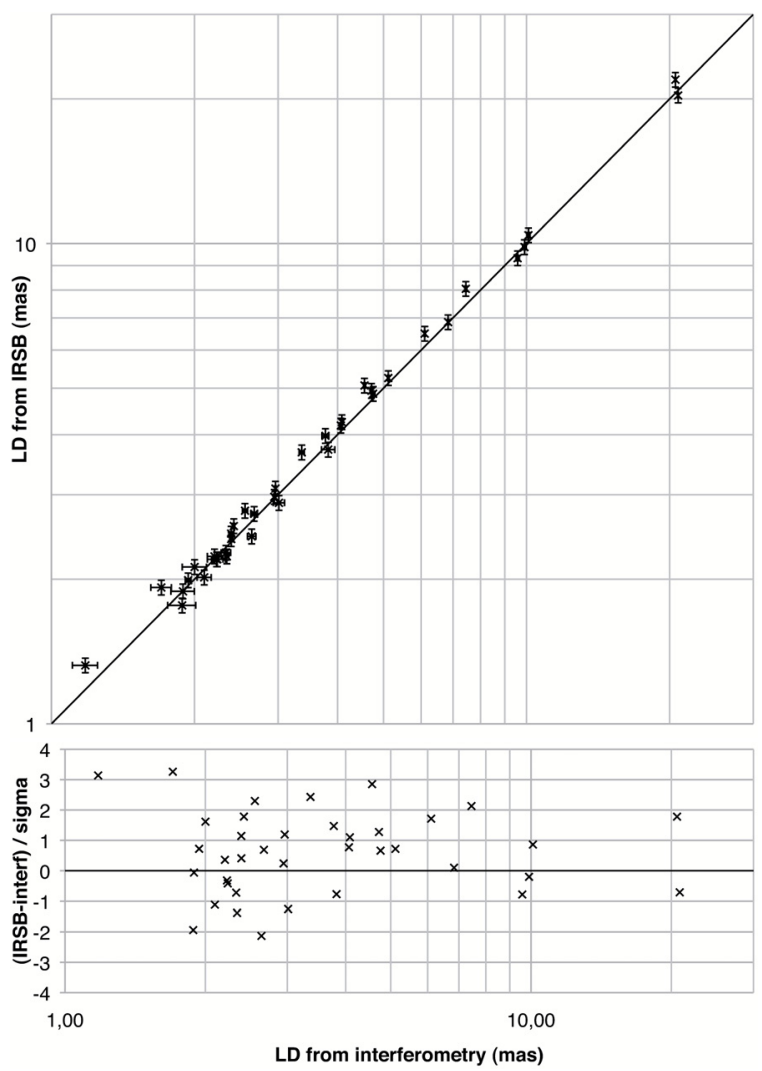

Fig. 1. Comparison of the limb-darkened angular diameters of the stars of our sample from direct interferometric measurements (horizontal axis) and photometric estimates based on the $F_{K}(V-K)$ infrared surface brightness-color relation (IRSB) from Kervella et al. (2004a). The lower plot shows the residuals as a function of the measured angular diameter.

These estimates were originally obtained by McWilliam (1990), Thévenin \& Idiart (1999), and Mallik (1998). Similarly, the $T_{\mathrm{eff}}$, metallicity, and luminosity of $\epsilon$ Oph are adapted from De Ridder et al. (2006) (also in agreement with Mazumdar et al. 2009). The bolometric luminosity was estimated using the $K$ band photometry and the corresponding bolometric corrections $B C(K)$ by Houdashelt et al. (2000). For $\gamma$ Sge, we extrapolated the $B C(K)$ value for $T_{\mathrm{eff}}=3805 \mathrm{~K}$ (Wittkowski et al. 2006). For $\epsilon$ Oph and $\delta$ Eri, we adopted the $T_{\text {eff }}$ metallicity and luminosity from De Ridder et al. (2006) and Thevenin et al. (2005), respectively. To derive the linear radius, we used the parallaxes from the Hipparcos catalogue (1997). We kept only the stars for which the relative uncertainty in the linear radius is smaller than $10 \%$. The distances to the selected stars range from 11 to $110 \mathrm{pc}$. Thanks to this proximity, we neglected the interstellar reddening for the computation of the bolometric luminosity.

Our final sample contains 38 giant and subgiant stars with spectral types from G5 to M0. We note that three stars in our sample have known asteroseismic oscillation frequencies: $\delta$ Eri, $\xi$ Hya, and $\epsilon$ Oph. This allowed an accurate determination of their masses by means of combining their radius and large frequency spacing (Thévenin et al. 2005; Mazumdar et al. 2009), which are estimated, respectively, to be $1.215,2.65$ (no error bars mentioned by the authors), and $1.85 \pm 0.05 M_{\odot}$.

\section{Convection length scales from the Sun}

The convection length scales can be calibrated using the Sun, as customarily done. We assume that $L_{\odot}=3.846 \times 10^{33} \mathrm{erg} \mathrm{s}^{-1}$ and 
$R_{\odot}=6.9599 \times 10^{10} \mathrm{~cm}$ and begin the solar evolution on the zero age main sequence. The calibration in luminosity, radius, and metal-to-hydrogen ratio $\frac{Z_{\text {surf }}}{X_{\text {surf }}}$ are achieved to better than $10^{-4}$ at the age of 4.6 Gyr for both MLT and CGM convection prescriptions. We consider the solar abundances and metal repartition of Asplund et al. (2005), hence $\frac{Z_{\text {surf }}}{X_{\text {surf }}}=1.65 \times 10^{-2}$. The microscopic diffusion is accounted for following Proffitt \& Michaud (1993). It induces a decrease in the helium and metal surface fractions in the course of the solar main-sequence.

In the MLT framework, we obtain $\alpha_{\text {mlt } \odot}=1.98$. The initial composition of the calibrated solar model is $X_{0}=0.7284$, $Y_{\mathrm{o}}=0.2578$, and $Z_{\mathrm{o}}=1.38 \times 10^{-2}$ and its solar age surface

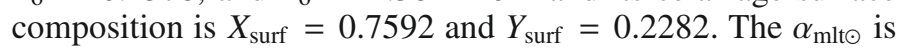
compatible with the estimate of VandenBerg et al. (2008) also using non-grey atmospheres as boundary conditions. Using the MARCS atmosphere models (Gustafsson et al. 2003), the latter authors find that $\alpha_{\text {mlt } \odot}=2.01$. Using the Kurucz atmosphere models (Kurucz 1993, see also Heiter et al. 2002), Samadi et al.

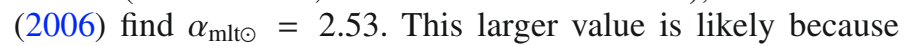
Samadi et al. begin interpolating between the atmosphere and interior gradients at the optical depth $\tau=4$, whereas we start at $\tau=1$ (see Sect. 2). The overadiabaticity induced by the atmosphere $\alpha=0.5$ extends over a larger region in their models and has to be compensated for by a more efficient convection at greater depth. We recall that $\alpha_{\text {mlt } \odot}$ depends on the assumed solar composition. For the composition of Grevesse \& Sauval (1998) $\left(\frac{Z_{\text {surf }}}{X_{\text {suf }}}=2.3 \times 10^{-2}\right)$, the solar calibration provides $\alpha_{\text {mlt॰ }}=1.67$.

In the CGM model framework, we obtain $\alpha_{\text {cgm } \odot}=0.77$. The initial composition of the calibrated CGM solar model is nearly the same as that of the calibrated MLT solar model. The value of $Z_{0}=1.38 \times 10^{-2}$ is identical while the hydrogen and helium fractions differ very slightly from those of the MLT case $X_{\mathrm{o}}=0.7280$ and $Y_{\mathrm{o}}=0.2582$, as do the current surface com-

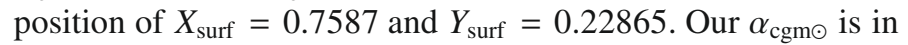
close agreement with the values of authors who used the same

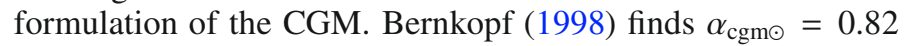
and Samadi et al. (2006) find $\alpha_{\text {cgm } \odot}=0.78$.

Theoretical atmosphere models probably account properly for the differential changes in the outer stellar regions with $T_{\text {eff }}$ and surface gravity. Nevertheless, in the solar case, empirical atmosphere relations seem to reproduce the limb darkening more accurately than theoretical ones (Blackwell et al. 1995). Following VandenBerg et al. (2008), we combined the advantages of theoretical and empirical $T(\tau)$ relations. To that extent, the values of $T(\tau)$ we used is the interpolated theoretical ATLAS12 relations $T_{\text {theory }}(\tau)$ rescaled by the empirical $T_{\mathrm{HM} 74}(\tau)$ relation for the solar atmosphere from Holweger \& Mueller (1974) as suggested by VandenBerg et al. (2008)

$T(\tau)=T_{\text {theory }}(\tau)+\frac{T_{\text {eff }}}{T_{\text {eff }, \odot}}\left[T_{\text {HM74 }}(\tau)-T_{\text {theory }, \odot}(\tau)\right]$.

If we introduce these semi-empirical atmosphere models, the solar calibration leads to a slightly larger $\alpha_{\text {cgm } \odot}=0.80$ for the CGM prescription. The initial and final solar surface compositions change negligibly. We discuss the influence of this semi-empirical atmosphere model in Sect. 5.3 below.

We give a word of caution on solar calibrations of convective length scales. They are as reliable as the solar models. The dynamics of the Sun radiation zone is largely uncertain as suggested by helioseismology (Turck-Chièze et al. 2004). It probably involves angular momentum transport through internal waves (Charbonnel \& Talon 2005; Mathis et al. 2008). Moreover, the mixing of the radiative interior associated with rotation (as in the tachocline) interacts with diffusion along the evolution (Brun et al. 1999). This changes the present-day surface composition. In this respect, we note that the calibration of the solar models using its recent composition determinations have led to helium content of the convection zone that systematically underestimate the measurement made thanks to seismology (Basu \& Antia 1995).

\section{Red giant branch calibrations}

\subsection{The cool edge of the RGB}

Depending on its mass, a red giant will not be at the same effective temperature for a given luminosity. In the case of open or globular clusters, all the objects on the RGB have approximately the same mass and the RGB is well defined. This is because clusters gather stars of the same age. No such assumption can be made for a sample that is representative of field stars in the solar neighbourhood: stars of different ages (and thus masses) are presumably on the RGB we consider.

It is however possible to set a lower limit to these masses. The limit is given by the age of the local Galactic disk and the evolutionary timescale of its low mass stars:

Age: for objects in the slightly subsolar metallicity range $(-0.25<[\mathrm{Fe} / \mathrm{H}]<-0.14)$, Liu \& Chaboyer (2000) suggest a maximum age of $11.7 \pm 1.9$ Gyr. In this study, we consider mainly models that have reached $10^{3} L_{\odot}$ on the RGB by that age as our RGB stars exhibits $[\mathrm{Fe} / \mathrm{H}]=-0.17$ on average. We can also set upper limits to the local Galactic disk age: it is probably younger than the oldest Galaxy globular clusters whose current age estimate is 12.6 Gyr (Krauss \& Chaboyer 2003) and certainly younger than the Universe $13.7 \pm 0.1$ Gyr (Komatsu et al. 2009).

Initial composition: The average metallicity of the 34 giants and four subgiants in Table 1 is $[\mathrm{Fe} / \mathrm{H}] \sim-0.17$. As is well known, the first dredge-up occurring near the base of the RGB erases most earlier diffusion effects such that the actual surface metal fraction is also the initial one. We set the initial helium content to be $Y=0.2582$, i.e., the amount of our solar-calibrated models. This choice could be criticized as the stellar nucleosynthesis simultaneously increases the metal and helium fractions and both of them appear (loosely) correlated (Fernandes et al. 1998). Furthermore, the estimate of the initial solar content helium remains a matter of debate as it is affected by dynamical phenomena in the solar interior (cf final caveat of Sect. 4). However, a discussion on helium would be irrelevant here because even a substantial change in its initial fraction has a negligible impact on the position of the RGB as we see shortly. In contrast, metals have a very strong influence on the effective temperatures and radii of RGB stars. Thus, in the following we repeat most of the calculations for the metallicities $[\mathrm{Fe} / \mathrm{H}]=-0.17$ and $[\mathrm{Fe} / \mathrm{H}]=0$. The fraction $Z=1.20 \times 10^{-2}$ of the solar metallicity models differs very slightly from our solarcalibrated models $Z=1.26 \times 10^{-2}$ because of the helium fraction difference between both sets. In Sect. 5.4, we performed additional calculations for $[\mathrm{Fe} / \mathrm{H}]=-0.34$ and computed models with compositions dedicated to the few stars in the sample with seismic data. The main compositions investigated are given in detail in Table 2.

Between $\sim 30$ and $\sim 300 L_{\odot}$, six stars clearly define the lower envelope of the local Galactic RGB in the classical HR diagram: $\sigma$ Gem, $\delta$ And, $\rho$ Boo, 91 Psc, 31 Leo, and $v$ Boo. Hereafter, we refer to them as "group I". Figure 2 displays them and also shows an object that is far off the general trend. This is $\delta^{1}$ Tau and is not considered in the following analysis. In the bolometric 


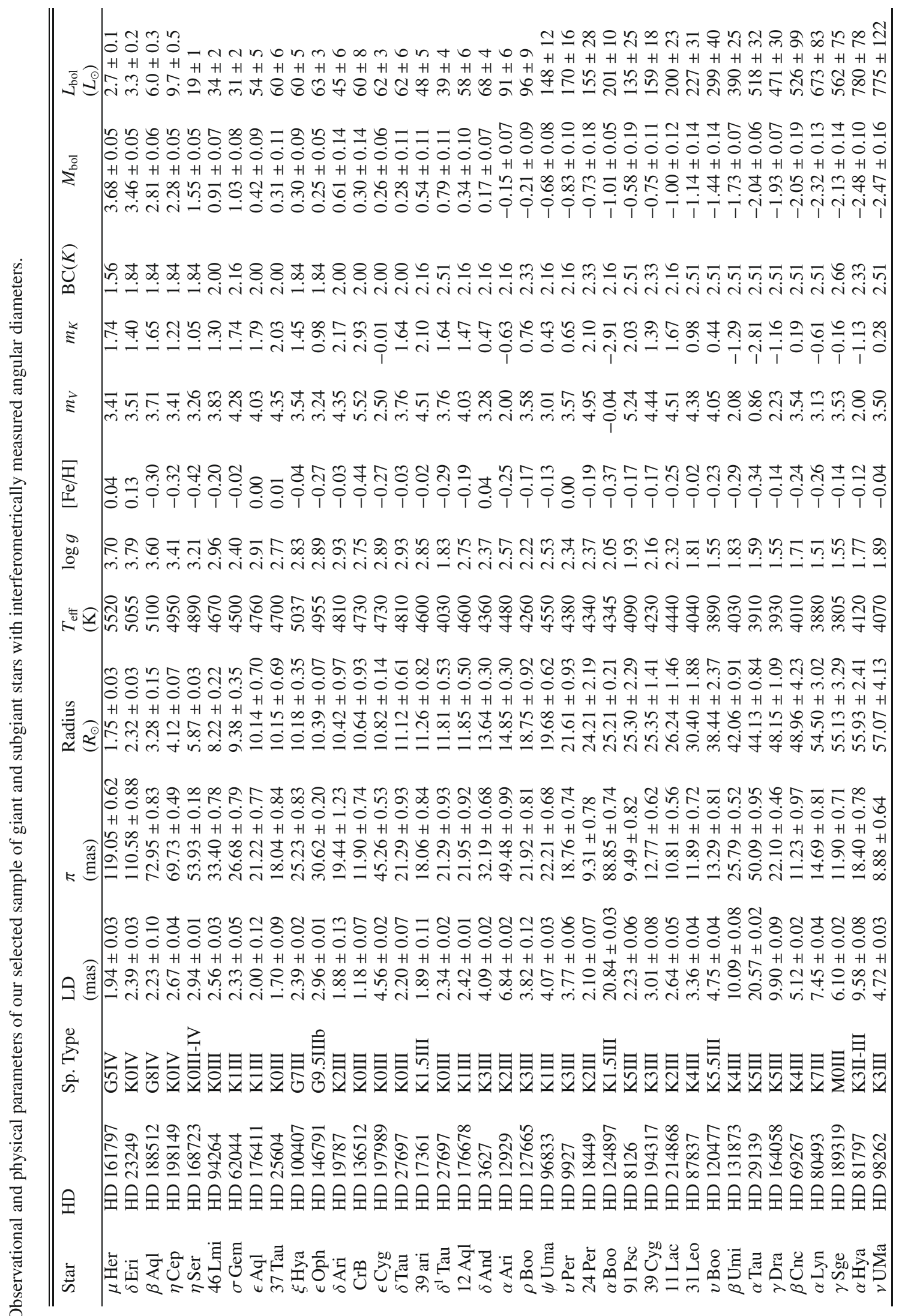


Table 2. The three main models in terms of composition.

\begin{tabular}{lcc}
\hline \hline$[\mathrm{Fe} / \mathrm{H}]$ & $\begin{array}{c}\text { Metals } \\
\text { mass fraction }\end{array}$ & $\begin{array}{c}\text { Hydrogen } \\
\text { mass fraction }\end{array}$ \\
\hline 0.0 & $1.20 \times 10^{-2}$ & 0.7297 \\
-0.17 & $8.18 \times 10^{-3}$ & 0.7336 \\
-0.34 & $5.55 \times 10^{-3}$ & 0.7362 \\
\hline
\end{tabular}

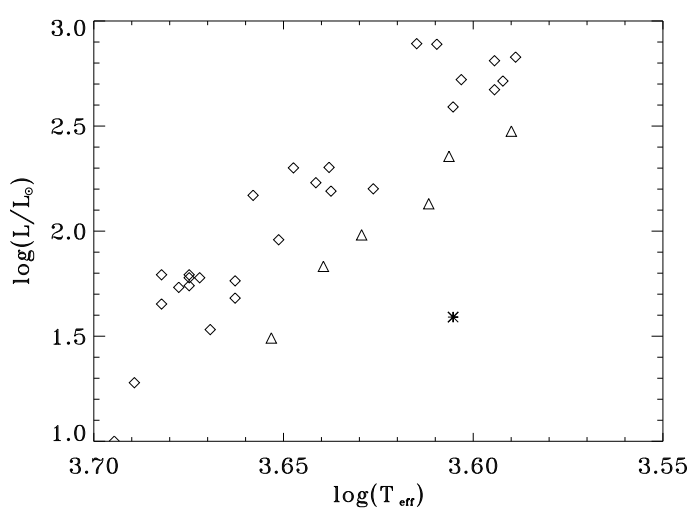

Fig. 2. Distribution of the current stellar sample in the HR diagram. Triangles: lower envelope of the RGB, see text for the names of the six corresponding objects. Diamonds: the other giants. Star: $\delta^{1}$ Tau.

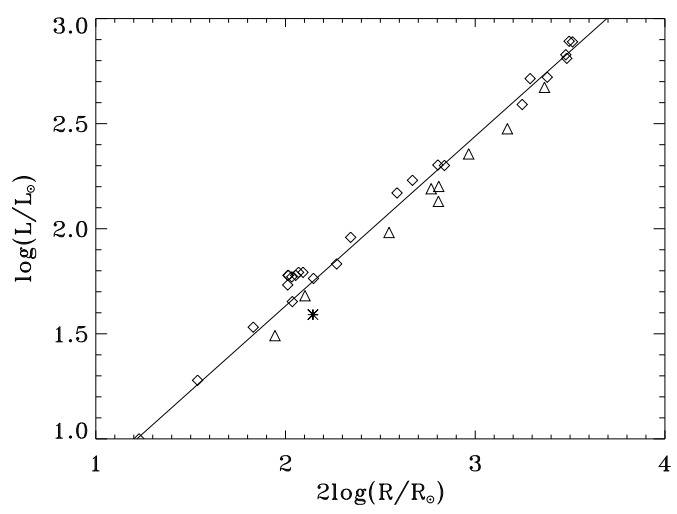

Fig. 3. Distribution of the current stellar sample in the log luminosity versus log squared radius diagram. The solid line is a linear fit to the sample by minimization of $\chi^{2}$. Triangles: group II, the nine giants having the largest radius with respect to the corresponding fitted radius of the sample. Diamonds: the other giants. Star: $\delta^{1}$ Tau.

luminosity versus stellar squared radius diagram, the lower envelope of the RGB is more difficult to see (Fig. 3). We selected the giants with the largest radii at a given luminosity in the following manner. We first performed a linear fit to the whole set of data. We then kept only the stars with the ten largest differences in radius from the fitted radius at similar luminosities: $\sigma$ Gem, 39 Ari, $\rho$ Boo, 24 Per, 91 Psc, 39 Cyg, 31 Leo, $v$ Boo, $\beta$ UMi, and $\gamma$ Dra. As in the case of the classical HR diagram, $\delta^{1}$ Tau was excluded from the sample as was $\beta \mathrm{UMi}$ because its metallicity is very low $([\mathrm{Fe} / \mathrm{H}]=-0.29)$ and we refer to the nine remaining stars as "group II". Out of the six stars forming the lower envelope of the RGB in the classical HR diagram, five also belong to the group of the nine most expanded objects. One could suggest that for simplicity, we rely also on the coolest stars in the subsequent analysis using the radii. We instead keep groups I and II. One purpose of this work is to use data on effective temperatures and radii independently: the radii are determined from

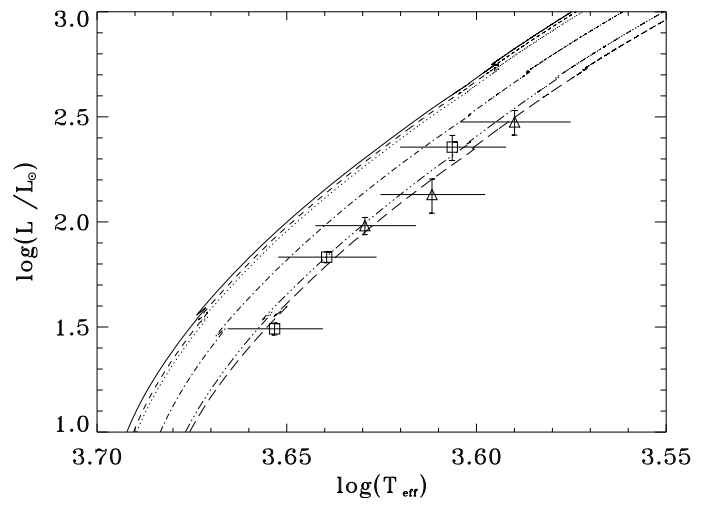

Fig. 4. RGBs in the HR diagram for models mlt1 to mlt6 of Table 3. The solid line is model mlt 1 , the dotted line is model mlt 2 , the dashed line is model mlt3, the dot-dashed line is model mlt4, the three-dot dashed line is model mlt5, and the long-dashed line is model mlt6. Models mlt 2 and mlt 3 show mass and helium fraction changes with respect to model mlt1. They are hardly discernable from this model. Model mlt4 shows the metallicity effect with respect to model mlt1. Only models mlt5 and mlt6 with lower $\alpha_{\text {mlt }}$ than the previous models fit the data satisfactorily. The data are the six giants of "group I" defined in the text and Fig. 2. Square symbols are for objects within 0.04 dex of solar metallicity, while triangles are for objects within $0.06 \operatorname{dex}$ of $[\mathrm{Fe} / \mathrm{H}]=$ -0.17 . The error bars in $\log \left(T_{\text {eff }}\right)$ are taken from McWilliam (1990), the error bars in log luminosity are adapted from Table 1.

interferometry, while the effective temperatures are determined from spectroscopy. Within the groups, the stars can be classified depending on the metallicity ${ }^{5}$. All of the stars $\sigma$ Gem, $\delta$ And, 31 Leo, 39 Ari are within 0.04 dex of the solar metallicity. All the other members of groups I and II are within 0.06 dex of $[\mathrm{Fe} / \mathrm{H}]=-0.17$ (and within 0.03 dex of this value if $v$ Boo is not considered). We now compare the data on these objects to the evolutionary tracks of models with different compositions, masses, and convection treatment length scales, first in the case of the MLT and then in the case of the CGM model.

\subsection{The cool edge of the RGB and the MLT}

Table 3 sums up the properties of the different models computed using the MLT, while Figs. 4 and 5 display their RGB evolutionary tracks. In the Table, Col. 1 is the model mass, Col. 2 the metallicity, Col. 3 the helium mass fraction, Col. 4 the age in Gyr at $10^{3} L_{\odot}$, and Col. 5 the $\alpha_{\text {mlt }}$. Columns 6 and 7 are the $\chi^{2}$ goodness of fit between the models and data, respectively, in the HR diagram and the radius luminosity diagram. The value of $\chi_{1}^{2}$ in Col. 6 is based on effective temperatures in stars of group I. It is defined by $\chi_{1}^{2}=\sum_{i=1}^{N} \frac{1}{N}\left[\frac{T_{\mathrm{eff}}^{\mathrm{mod}}-T_{\mathrm{eff}}^{\mathrm{obs}}}{\Delta T_{\mathrm{eff}}^{\mathrm{obs}}}\right]^{2}$, where $T_{\mathrm{eff}}^{\mathrm{obs}}$ and $T_{\mathrm{eff}}^{\mathrm{mod}}$ are, respectively, the observed effective temperature of an object and that of the model corresponding to the same luminosity as the object. The catalog of Cayrel de Strobel et al. (2001) contains mostly the values of $T_{\text {eff }}$ from McWilliam (1990). Accordingly, our $\Delta T_{\mathrm{eff}}^{\mathrm{obs}}$ are adapted from this author to be $\Delta T_{\mathrm{eff}}^{\mathrm{obs}}=130 \mathrm{~K}$. In Col. $7 \chi_{2}^{2}$ is computed from the radii of the nine stars of group II according to $\chi_{2}^{2}=\sum_{i=1}^{N} \frac{1}{N}\left[\frac{R_{\mathrm{mod}}^{2}-R_{\mathrm{obs}}^{2}}{\Delta R_{\mathrm{obs}}^{2}}\right]^{2}$. We do not consider the traditional $\chi^{2}$ formula but the reduced $\chi^{2}$ to allow a comparison of

5 We could not find the error bars in the metallicities of group I and II stars in the catalog of Cayrel de Strobel (2001). We just mention that the error bars, when present in this catalog, are generally around 0.1 dex. 


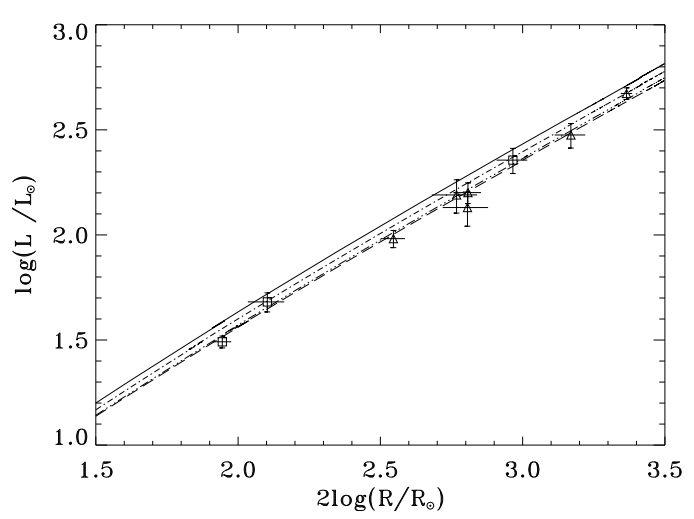

Fig. 5. RGBs in a bolometric luminosity vs. stellar squared radius diagram for models mlt1, mlt4, mlt5, and mlt6 of Table 3 . The lines conventions are similar to those in Fig. 4. Models mlt2 and mlt 3 are not shown as they could hardly be distinguished from the track of model mlt1. The data are the nine giants of group II defined in text. As in Fig. 4, square symbols are for objects within 0.04 dex of solar metallicity, triangles represent objects within $0.06 \mathrm{dex}$ of $[\mathrm{Fe} / \mathrm{H}]=-0.17$. The best fits are obtained for the models mlt5 and mlt6.

the fits when the number of stars is changed. We can see that changing the sample of stars from group I to group II hardly changes the goodness of the fit. Columns 6 and 7 are based on the stars within 0.06 dex of the metallicity given in Col. 2 for consistency between the composition of the models and the observations (the nearly solar-metallicity objects over groups I and II are $\sigma$ Gem, $\delta$ And, 31 Leo, and 39 Ari). However, the $\chi^{2}$ in parentheses in Cols. 6 and 7 relate the models to all the members of group I and II, respectively. Finally, Col. 8 is the model name and bold fonts indicate modelling input changes with respect to the (reference) model mlt1.

The following observations can be made:

i) The model mlt1 uses the solar-calibrated $\alpha_{\text {mlt }}$ and reaches $10^{3} L_{\odot}$ at $\approx 11.5 \mathrm{Gyr}$ i.e. at nearly the age estimated for the local Galactic disk. Its RGB track does not fit the six coolest objects within their observational error bars. The models mlt 2 and mlt 3 are, respectively, less massive and helium poorer than the model mlt1. Model mlt 2 is extreme in the sense that it reaches $10^{3} L_{\odot}$ at $\approx 13.9 \mathrm{Gyr}\left(\right.$ or $10^{2} L_{\odot}$ at $\approx 13.88 \mathrm{Gyr}$ ), which is older than the current age estimate of the Universe of $13.7 \pm 0.12$ Gyr (Komatsu et al. 2009). Model mlt3 is also extreme in the sense that its helium fraction is that of Big Bang nucleosynthesis (Coc et al. 2004) and evidently cannot be lowered any further. Both models mlt 2 and mlt 3 are in slightly closer agreement with the data than model mlt 1 and are almost undistinguishable from it in Fig. 4. They demonstrate that acceptable changes in mass or helium fraction can hardly improve the poor fit of model mlt1.

ii) Even though group I corresponds to $[\mathrm{Fe} / \mathrm{H}] \approx-0.1$ on average, one can distinguish two metallicity subgroups because $\sigma \mathrm{Gem}, \delta$ And, and 31 Leo are nearly of solar metallicity, while $\rho$ Boo, $91 \mathrm{Psc}$, and $v$ Boo are within 0.06 dex of $[\mathrm{Fe} / \mathrm{H}]=-0.17$ (see Table 1 ). It is necessary to investigate the metallicity effects. The solar metallicity $0.95 M_{\odot}$ model mlt4 provides a better fit to the observations than the previous ones: its $\chi_{1}^{2}$ fit to the whole group I is 0.95 and to the subsample of the three solar metallicity stars is 0.72 . Together with models mlt $2 \&$ mlt3, this model should also be considered as extreme because of its age at $10^{3} L_{\odot}: 13.7 \mathrm{Gyr}$.
The increase in metal fraction lowers the luminosity and thus decelarates the evolution with respect to the same mass model mlt1.

iii) Models with lower $\alpha_{\text {mlt }}$ than the solar value provide much better fits to the data. For the model mlt5 $\left(\alpha_{\mathrm{mlt}}=1.68\right.$, $0.95 M_{\odot}$, and $\left.[\mathrm{Fe} / \mathrm{H}]=-0.17\right) \chi_{1}^{2}=0.16$. The model reaches $10^{3} L_{\odot}$ at $11.5 \mathrm{Gyr}$. The model mlt6 $\left(\alpha_{\mathrm{mlt}}=1.68\right.$, $1.13 M_{\odot}$, and $[\mathrm{Fe} / \mathrm{H}]=0$ ) has $\chi_{1}^{2}=0.078$ and reaches $10^{3} L_{\odot}$ at 7.5 Gyr. The pattern of the goodness of the fit is similar if one considers the luminosity vs. square radius instead of the HR diagram. The $\chi_{2}^{2}$ and $\chi_{1}^{2}$ have the same orders of magnitudes and variations for models mlt 1 to mlt 4 . The two best fit solution values of $\chi_{2}^{2}$ are also obtained for models mlt6 and mlt5 with 0.54 and 1.1 , respectively. In these cases, however the $\chi_{2}^{2} \mathrm{~s}$ remain larger than the $\chi_{1}^{2} \mathrm{~s}$. This is mostly due to the errorbars in square radius being smaller than the errorbars in effective temperatures (see Figs. 4 and 5).

iv) Studies have suggested that the models with a solarcalibrated $\alpha_{\text {mlt }}$ can properly describe the RGB (Alonso et al. 2000; Ferraro et al. 2006; VandenBerg et al. 2008). It is likely that our confilcting conclusion stems from the systematic use of non-grey atmospheres with a low $\alpha_{\mathrm{mlt}}=0.5$ parameter. VandenBerg et al. (2008) rely on the MARCS atmosphere models where they assume the high value $\alpha_{\mathrm{mlt}}=1.80 \mathrm{of}$ the interior. Alonso et al. (2000) and Ferraro et al. (2006) both rely on the Krishna Swamy (1966) $T-\tau$ atmosphere relation but they do not mention the optical depth $\tau_{\mathrm{b}}$ at which they connect the atmosphere to the interior. In any case, the absence of a low efficiency surface convection region can be criticized. $\tau_{\mathrm{b}} \approx 1$ would mean that the outermost convective regions are handled with the interior $\alpha_{\text {mlt }}$ parameter, which is not supported by the Balmer line profiles (see Sect. 2). On the other hand, $\tau_{\mathrm{b}} \geq 10$ would imply that the KrishnaSwamy $\mathrm{T}-\tau$ relation is extended down to the convective regime, which is incorrect as mentioned by Krishna Swamy (1966).

\subsection{The cool edge of the RGB and the CGM model}

The MLT is widely used in stellar modelling. However, the FST approach initially developed by Canuto \& Mazzitelli (1991) is physically more consistent (Mazzitelli 1999). When compared to observations, it seems to provide a better description of stellar convection as supported by many studies in seismology (Basu \& Antia 1994; Monteiro et al. 1996; Samadi et al. 2006) and stellar evolution (Stothers \& Chin 1997; Montalban et al. 2001). For this reason, it is important to measure any changes in the characteristic length scale of the FST model. In a more appropriate version of this model, the characteristic length scale is the distance to the convectively stable region, and thus is not a free parameter. This approach is not implemented in our stellar evolution code because it would require substantial changes to compute in the same iterations both the convection efficiency and distance to the convectively stable region. We instead use the Canuto et al. (1996) version (CGM) of the FST with a characteristic length scale $\alpha_{\mathrm{cgm}} H_{\mathrm{p}}$ as mentioned in the previous section. The precise description of the version of the model including the parameters is given in Appendix B.

Table 4 gathers the properties of the models made using the CGM model, while Figs. 6 and 7 display their RGB evolutionary tracks. Columns conventions are the same in Tables 4 and 3. We do not report here the models exploring the effects of a change in mass or helium fraction (corresponding to models mlt $2 \&$ mlt 3 
Table 3. Characteristics of the RGB models using the MLT.

\begin{tabular}{lcccccccc}
\hline \hline$M / M_{\odot}$ & {$[\mathrm{Fe} / \mathrm{H}]$} & $Y$ & Age at $10^{3} L_{\odot}$ & $\alpha_{\text {mlt }}$ & $\chi_{1}^{2}$ & $\chi_{2}^{2}$ & Model name \\
\hline 0.95 & -0.17 & 0.2582 & 11.5 & 1.98 & $3.8(3.2)$ & $4.0(3.8)$ & mlt1 \\
$\mathbf{0 . 9 0}$ & -0.17 & 0.2582 & 13.9 & 1.98 & $3.0(2.5)$ & $3.2(3.1)$ & mlt2 \\
0.95 & -0.17 & $\mathbf{0 . 2 4 8 2}$ & 12.3 & 1.98 & $3.4(2.8)$ & $3.6(3.4)$ & mlt3 \\
$\mathbf{0 . 9 5}$ & $\mathbf{0 . 0 0}$ & 0.2582 & 13.7 & 1.98 & $0.72(0.95)$ & $1.5(1.4)$ & mlt4 \\
0.95 & -0.17 & 0.2582 & 11.5 & $\mathbf{1 . 6 8}$ & $0.16(0.11)$ & $1.1(0.91)$ & mlt5 \\
1.13 & $\mathbf{0 . 0 0}$ & 0.2582 & 7.5 & $\mathbf{1 . 6 8}$ & $0.078(0.070)$ & $0.54(1.3)$ & mlt6 \\
\hline
\end{tabular}

Table 4. Characteristics of the RGB models using the CGM.

\begin{tabular}{lccccccc}
\hline \hline$M / M_{\odot}$ & {$[\mathrm{Fe} / \mathrm{H}]$} & $Y$ & Age at $10^{3} L_{\odot}$ & $\alpha_{\text {cgm }}$ & $\chi_{1}^{2}$ & $\chi_{2}^{2}$ & Model name \\
\hline 0.95 & -0.17 & 0.2582 & 11.6 & 0.77 & $6.1(5.1)$ & $7.1(6.2)$ & cgm1 \\
$\mathbf{0 . 9 5}$ & $\mathbf{0 . 0 0}$ & 0.2582 & 13.8 & 0.77 & $1.1(1.6)$ & $1.8(2.3)$ & cgm2 \\
0.95 & -0.17 & 0.2582 & 11.8 & $\mathbf{0 . 6 2}$ & $0.21(0.11)$ & $0.70(0.57)$ & cgm3 \\
1.17 & $\mathbf{0 . 0 0}$ & 0.2582 & 6.9 & $\mathbf{0 . 6 2}$ & $0.057(0.077)$ & $0.40(0.76)$ & cgm4 \\
$0.95^{\dagger}$ & -0.17 & 0.2582 & 11.5 & $\mathbf{0 . 8 0}$ & $7.4(6.1)$ & $9.1(7.8)$ & cgm5 \\
\hline
\end{tabular}

Notes. Bold fonts indicate inputs changes with respect to the (reference) model mlt 1 and cgm1. The model cgm5 mentioned by $\dagger$ is built with the semi-empirical atmosphere described in Sect. 4.

in 5.2). Similar changes in these parameters alter the location of the RGB as little by as in the case of the MLT.

The following comments can be made:

i) Models cgm1 and mlt1 are identical except for their atmosphere boundary conditions and convection prescriptions. They are both affected by the same drawback as the RGBs they define are too warm with respect to the observations. Model cgm1 RGB is even warmer than that of model mlt1. This is a consequence of the higher efficiency of adiabatic convection in the CGM prescription than in the MLT. In the case of deep convective envelopes, this produces higher effective temperature objects (Mazzitelli et al. 1995). As can be seen in Fig. 6, for higher luminosities (i.e. for larger convective envelopes) the difference in effective temperatures between models mlt1 and cgm1 becomes larger.

ii) Having a solar metallicity, the model $\operatorname{cgm} 2$ is 0.17 dex more metal-rich than the model cgm1. However, in spite of its lower effective temperature and larger radius it also fails to fit the corresponding observations. As for the mlt models, the $\chi^{2}$ fits in Table 4 are related to the subgroups of stars exhibiting the same metallicity as the models. For the $[\mathrm{Fe} / \mathrm{H}]=0$ models, only $\sigma \mathrm{Gem}, \delta$ And, and 31 Leo are considered within group I, while only $\sigma$ Gem, 31 Leo, and 39 Ari are considered within group II. Models with masses lower than cgm 2 and the same metallicity could in principle fit the data. However, with an age over $13.8 \mathrm{Gyr}$ at $10^{3} \mathrm{~L}_{\odot}$, model cgm2 sets a lower limit to the mass.

iii) Both models cgm 1 and cgm 2 have the solar-calibrated $\alpha_{\text {cgm }}$ value. The sub-solar $\alpha_{\text {cgm }}$ models cgm 3 and cgm 4 produce a better agreement to the selected samples of stars in the two considered diagrams (Figs. 6 and 7) as can also be seen in Table 4. The model cgm 3 has $[\mathrm{Fe} / \mathrm{H}]=-0.17$ and $0.95 M_{\odot}$, while cgm 4 has $[\mathrm{Fe} / \mathrm{H}]=0$ and $1.17 M_{\odot}$. Thus models cgm3 and cgm 4 correspond, respectively, to models mlt5 and mlt6 in terms of masses and metallicities.

iv) In an attempt to investigate the atmosphere modelling effects, the model cgm5 was built with the semi-empirical approach for the atmosphere calculation described in Sect. 4. Accordingly, its $\alpha_{\text {cgm }}$ is 0.80 which is the solar convective scale when the semi-empirical atmosphere are used. The cgm5 RGB lies very close to the cgm1 RGB and is therefore too warm to fit the lower envelope of the observations. Models cgm1 and cgm5 illustrate that a change to semiempirical atmospheres does not reduce the gap between calculations and observations.

\subsection{The global RGB and the seismic targets}

We now intend to use the remaining data we have about the sample of 38 stars. First, we check how the stars are distributed between the evolutionary tracks of different masses. We consider the two surface convection prescriptions with convective length scales calibrated on either the Sun or the lower envelope of the current RGB in the preceding section.

Assuming that the stellar mass distribution of our sample is the present-day Galactic field stellar mass function (hereafter PDMF), the question we can investigate is whether the distribution of the sample between the evolution tracks of different masses agrees with the PDMF. The use of the PDMF relies here on two assumptions. First, it assumes that the mass loss of the preceding main-sequence and giant branch evolution is negligible. There is no indication of significant mass loss before the RGB tip. When using the formula advocated in Catelan (2000), we find that the $1.5 M_{\odot}$ model looses less than $0.005 M_{\odot}$ before reaching $8 \times 10^{2} L_{\odot}$. For higher masses, the mass loss is lower. Second, it assumes that the stars of our sample are a well-mixed Galactic field population that formed in a variety of star-forming regions and environments. Should a substantial fraction of the stars in our sample be formed in a single protocluster star forming region, deviations, in both the slope and the characteristic mass, between the PDMF and that of our sample may be expected (Dib et al. 2010). The observed PDMF of stars in the local Galactic field is well fitted by a multi-exponent power-law functional form (Kroupa 2002 and 2007)

$$
\begin{aligned}
& \frac{\mathrm{d} N}{\mathrm{~d} M}=k(0.5 / 0.08)^{-1.3}(M / 0.5)^{-2.3} \text { if } 0.5<M / M_{\odot}<1.0 \\
& \frac{\mathrm{d} N}{\mathrm{~d} M}=k(0.5 / 0.08)^{-1.3}(1.0 / 0.5)^{-2.3}(M)^{-2.7} \text { if } M / M_{\odot}>1 .
\end{aligned}
$$

In Eqs. (2) and (3), $k=0.877$ and $N$ is the density of stars of mass $\mathrm{M}$ (in solar mass units). The corresponding repartition below, between, and above $1.5 M_{\odot}$ and $2.5 M_{\odot}$ is given in the 


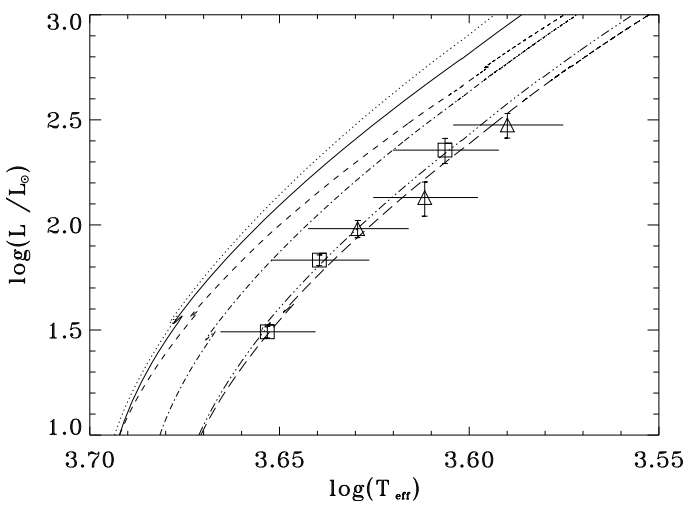

Fig. 6. RGBs in the HR diagram for models cgm1 to cgm 5 of Table 4. The track of model mlt 1 of Table 3 is also given for comparison. The solid line is model cgm1, the dashed line is model mlt1, the dot-dashed line is model cgm2, the three-dot-dashed line is model cgm 3 and the long dashed line is model cgm4. The dotted line is model cgm5. The data are the six giants of group I defined in the text and Fig. 2. Following Fig. 4 the squares and triangles respectively stand for solar and subsolar metallicity objects. Only models cgm 3 and cgm 4 with lower $\alpha_{\text {cgm }}$ than the other models fit the data satisfactorily.

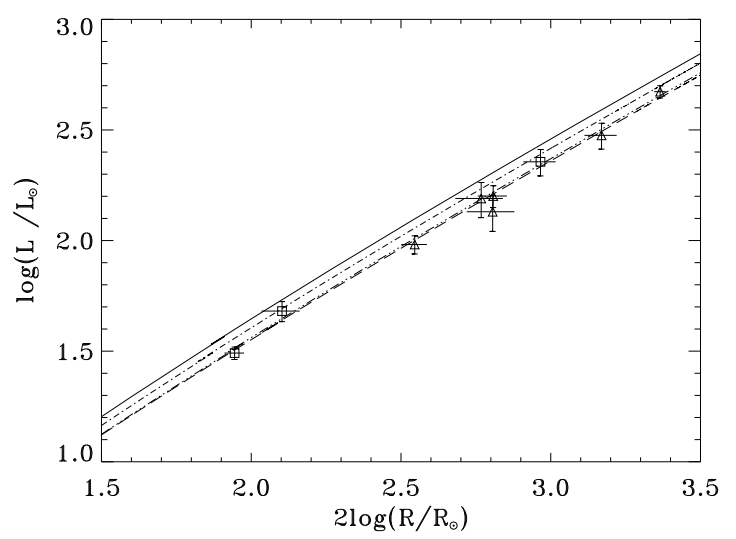

Fig. 7. RGBs in a bolometric luminosity vs. stellar squared radius for models cgm1 to cgm4 of Table 4 . The lines conventions are similar as in Fig. 6. The data are the nine giants of group II defined in the text (see also Fig. 3). The data symbols are the same as in Fig. 5: triangle symbols are for objects within 0.04 dex of solar metallicity, triangles for objects within $0.06 \mathrm{dex}$ of $[\mathrm{Fe} / \mathrm{H}]=-0.17$.

second column of Table 5. We refer to it as the "truncated" PDMF as we derive the distribution using the previous power laws and by making the hypothesis that no star has a mass lower than $0.95 M_{\odot}$, which we found to be the mass of the objects forming the lower envelope of the RGB (see Sect. 5.2). If we had considered a lower truncature mass such as $0.90 M_{\odot}$ (corresponding to an unrealistic age for the oldest stars of the sample), the distribution would hardly have been affected. As is well known, the position of the RGB depends strongly on the metallicity. For instance, for the $1.5 M_{\odot} \mathrm{CGM}$ models at $10^{2} L_{\odot}$, a change from $[\mathrm{Fe} / \mathrm{H}]=-0.34$ to $[\mathrm{Fe} / \mathrm{H}]=0$ lowers the $T_{\text {eff }}$ by $240 \mathrm{~K}$ (and, respectively, increases the radius from 15.8 to $17.7 R_{\odot}$ ). Thus, the distribution of stars relative to the evolutionary tracks is made with models having nearly the same metallicity as the observed stars. We compare data and models for three metallicities. The $[\mathrm{Fe} / \mathrm{H}]=-0.34$ tracks are used for objects exhibiting $[\mathrm{Fe} / \mathrm{H}]<-0.25$. The $[\mathrm{Fe} / \mathrm{H}]=-0.17$ tracks are used to estimate the distribution of objects having $-0.25 \leq[\mathrm{Fe} / \mathrm{H}] \leq$ -0.08 . Finally, the $[\mathrm{Fe} / \mathrm{H}]=0$ tracks are used when the observation gives $[\mathrm{Fe} / \mathrm{H}]>-0.08$.

The repartition between the tracks has been drawn from the luminosity vs. stellar squared radius diagram (see Fig. 8). It is reported in Table 5 for the different assumptions about the convective length scales. Column 3 shows the distribution for stars with $\alpha_{\mathrm{mlt}}=1.98$, Col. 4 for stars with $\alpha_{\mathrm{mlt}}=1.68$, Col. 5 for stars with $\alpha_{\text {cgm }}=0.77$, and Col. 6 for stars with $\alpha_{\text {cgm }}=0.62$. For a given mass, composition, and luminosity, the larger the characteristic convective length scale the warmer the model. Being warmer, the solar-calibrated models $\left(\alpha_{\text {mlt }}=1.98\right.$ or $\left.\alpha_{\text {cgm }}=0.77\right)$ appear to delineate a local RGB strongly biased toward lower mass stars. The models calibrated on the lower envelope of the RGB $\left(\alpha_{\text {mlt }}=1.68\right.$ or $\left.\alpha_{\text {cgm }}=0.62\right)$ are in better agreement with the PDMF. This confirms the slightly less efficient CGM convection in the RGB regime found in Sect. 5.3. However, this trend is merely indicative. The small number of stars in each mass bins causes large statistical uncertainties. Moreover, the RGB tracks of stars with convective cores on the main sequence depend on the amount of overshooting during this phase. This amount of core overshooting is not a well-established quantity. The larger the overshooting the more massive the helium cores, and the smaller the radius at a given luminosity. According to the mass dependence of core overshooting of Claret 2007 (see Fig. 13 of this author), we assumed that $\alpha_{\mathrm{ov}}=0.1 H_{\mathrm{p}}$ and $\alpha_{\mathrm{ov}}=0.2 H_{\mathrm{p}}$ in the $1.5 M_{\odot}$ and $2.5 M_{\odot}$ models, respectively.

We dedicated special attention to $\delta$ Eri, $\xi$ Hya, and $\epsilon$ Oph as asteroseismology has allowed an accurate determination of their masses. Our $\delta$ Eri models have a mass of $1.215 M_{\odot}$ (Thévenin et al. 2005) and $X=0.7256$ to account for the star's metallicity $[\mathrm{Fe} / \mathrm{H}]=0.13$. The $\epsilon$ Oph models have a mass of $1.85 M_{\odot}$ (Mazumdar et al. 2009) and $X=0.7326([\mathrm{Fe} / \mathrm{H}]=-0.12)$. Finally, the $\xi$ Hya models have a mass of $2.65 M_{\odot}$ (Thévenin et al. 2005) and a hydrogen mass fraction $X=0.7308([\mathrm{Fe} / \mathrm{H}]=$ $-0.04)$. All of these models have the same helium mass fraction $Y=0.2582$. We assume a convective core overshooting of $0.1 H_{\mathrm{p}}$ in $\delta$ Eri and $0.2 H_{\mathrm{p}}$ in both $\xi$ Hya and $\epsilon$ Oph. Figure 9 shows that the position of the $\delta$ Eri models depends weakly on the chosen convection length scale. The $\alpha_{\text {cgm }}=0.77$ and $\alpha_{\text {mlt }}=1.98$ (solar-calibrated) models cannot be ruled out as they fit the data in the errorbars. However, the $\alpha_{\text {cgm }}=0.62$ and $\alpha_{\mathrm{mlt}}=1.68$ models converge right to the observed radius at the observed luminosity! For $\xi$ Hya, only the $\alpha_{\text {cgm }}=0.62$ and $\alpha_{\text {mlt }}=1.68$ models fit the observations within the errorbars in Fig. 10. In contrast, the $\epsilon$ Oph tracks with the small value of $\alpha_{\text {cgm }}=0.62$ very interestingly do not fit them. This might be because $\epsilon$ Oph is not on its first ascent of the RGB but a "red clump" star i.e. in the helium core burning phase. There are several indications of this. First $\epsilon$ Oph belongs to the compact group of six stars that can be seen at $\log \left(L / L_{\odot}\right) \approx 1.7$ in Fig. 3 and that strongly suggest the red clump. The second indication is provided by the Mazumdar et al. (2009) models. They spend 20 times more time in the $\epsilon$ Oph errorbox in the HR diagram during the helium core burning stage than during the first ascent of the RGB, which means that we are far more likely to observe a helium burning $\epsilon$ Oph than one on its first ascent ${ }^{6}$. Finally, except for its mass, $\epsilon$ Oph seems identical to $\xi$ Hya, which is likely to be on its first ascent of the RGB. Their metallicities only differ by 0.08 dex. Their luminosities (5\% difference), radii (2\%

\footnotetext{
${ }^{6}$ Our stellar evolution code cannot pass the so-called helium flash and follow the subsequent evolution of stars lighter than $\approx 2.1 M_{\odot}$ such as $\epsilon$ Oph during their helium core-burning phase.
} 
A\&A 526, A100 (2011)

Table 5. Mass repartition from the PDMF and for the MLT and CGM models of convection.

\begin{tabular}{lccccc}
\hline \hline Mass range & Truncated & $\alpha_{\mathrm{mlt}}$ & $\alpha_{\mathrm{mlt}}$ & $\alpha_{\mathrm{cgm}}$ & $\alpha_{\mathrm{cgm}}$ \\
& PDMF & 1.98 & 1.68 & 0.77 & 0.62 \\
\hline$M<1.5 M_{\odot}$ & $54 \%$ & $76 \pm 4 \%$ & $54 \pm 13 \%$ & $84 \pm 15 \%$ & $49 \pm 11 \%$ \\
$1.5 M_{\odot} \leq M \leq 2.5 M_{\odot}$ & $27 \%$ & $24 \pm 8 \%$ & $41 \pm 11 \%$ & $16 \pm 7 \%$ & $40 \pm 10 \%$ \\
$M>2.5 M_{\odot}$ & $19 \%$ & $0 \%$ & $5 \pm 4 \%$ & $0 \%$ & $11 \pm 5 \%$ \\
\hline
\end{tabular}

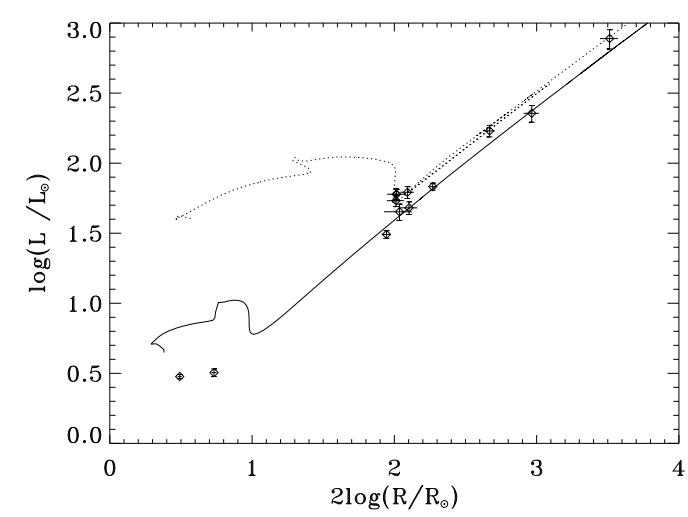

Fig. 8. Example of RGBs in the bolometric luminosity vs. stellar squared radius diagram for models using the CGM convection model and having $[\mathrm{Fe} / \mathrm{H}]=0$ where $1.5 M_{\odot}$ is given by the solid line and $2.5 M_{\odot}$ the dashed line. The evolutionary paths are shown from the zero age main sequence. For the sake of clarity, only the stars with $[\mathrm{Fe} / \mathrm{H}]>-0.08$ are displayed.

difference), and effective temperatures ( $80 \mathrm{~K}$ difference) are the same within errorbars but the masses differ strongly. This difference induces a difference in model radii of $\approx 15 \%$ at $\approx 60 L_{\odot}$ if both stars are on the RGB and the same convective length scale is considered. In contrast to $\epsilon \mathrm{Oph}, \xi$ Hya is probably not in the helium burning stage. We found the minimum luminosity of the $2.65 M_{\odot}$ models during the core helium burning (eAGB) around $80 L_{\odot}$. This value, in agreement with stellar grid calculations (Schaller et al. 1992), stands well outside the luminosity errorbar for $\xi$ Hya. Finally, apart from $\epsilon$ Oph, the seismic data combined with radii measurements suggest a drop in the convective length scale confirming the previous results. The seismic targets are in small number at the moment and Figs. 9 and 10 mostly illustrate that combining seismologically determined masses with interferometric radii provides very sensitive tests of the surface convection efficiency. In the near future, seismology should allow many more accurate mass and radius determinations (Basu et al. 2010).

\section{Discussion}

This work aims to constrain the outer convection prescriptions of low-mass red giants and subgiants by using their absolute luminosities, effective temperatures, interferometric measurements of their radii and seismic data. The observational sample consists of 38 Galactic disk nearby stars. It was selected on the basis of interferometric radii measured with a better than 10 percent accuracy. The average metallicity of the sample is $[\mathrm{Fe} / \mathrm{H}]=-0.17$. There are small but significant metallicity differences between the stars. Age and mass differences are also expected. From the modelling point of view, we used a modified version of the secular stellar evolution code CESAM. Special care was devoted to the atmosphere boundary conditions. We computed two

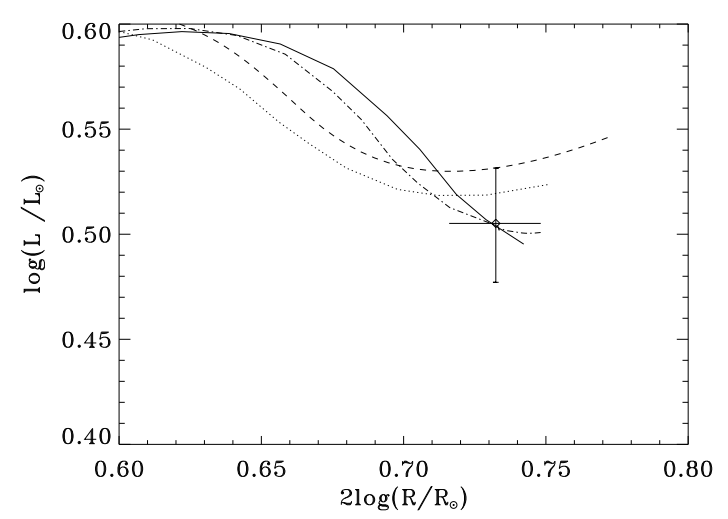

Fig. 9. $\delta$ Eri evolution tracks for $\alpha_{\text {cgm }}=0.62$ (solid line) $\alpha_{\text {cgm }}=0.77$ (dotted line), $\alpha_{\mathrm{mlt}}=1.68$ (dot-dashed line), and $\alpha_{\mathrm{mlt}}=1.98$ (dotted line). All models match the observed radius at the observed luminosity within the errorbars. However, the lower convective length scales models $\alpha_{\mathrm{cgm}}=0.62$ and $\alpha_{\mathrm{mlt}}=1.68$ provide a closer fit to the data.

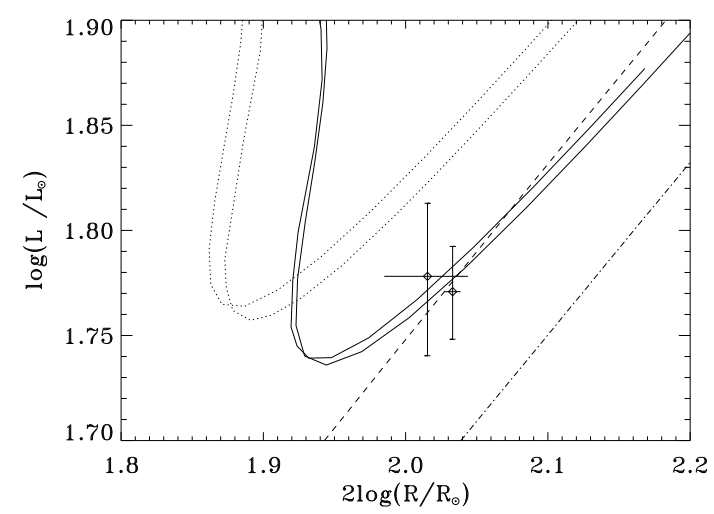

Fig. 10. Solid lines show the evolution tracks for $\alpha_{\mathrm{cgm}}=0.62$ and $\alpha_{\mathrm{mlt}}=$ 1.68 and a mass of $2.65 M_{\odot}$. The dotted lines correspond to the same mass but $\alpha_{\text {cgm }}=0.77$ and $\alpha_{\text {mlt }}=1.98$. The solar-calibrated models for convection do not fit the observations. Dashed line and dot-dashed lines are $1.85 M_{\odot}$ models, respectively, for $\alpha_{\text {cgm }}=0.77$ and $\alpha_{\text {cgm }}=0.62$. The smaller radius object is $\xi$ Hya and the larger one $\epsilon \mathrm{Oph}$.

grids of non-grey atmospheres surrounding the expected surface gravities and effective temperatures. The first grid, based on the PHOENIX/1D calculations, relies on the mixing length theory for the treatment of convection, while the second grid, based on the ATLAS12 calculations, relies on a modified version of the full spectrum of turbulence model (see Appendix B). We considered the boundary conditions of the interior model at the Rosseland optical depth 20 . In the regime of surface conditions encountered in red giants, superadiabatic convection extends above and below this limit. The same model of convection was used for the atmosphere and the interior but different convection length scales were adopted in the two regions. A procedure of linear interpolation of the thermal gradient with the optical 
depth allows a smooth transition between them. Other important changes to CESAM were made to enable the modelling of late stages of stellar evolution (see Appendix A). We chose the Asplund et al. (2005) solar repartition for metals in the opacity tables. The total metallicity was varied to account for solar or slightly subsolar metal content in the sample and the variations between the stars in dedicated cases.

We proceeded in three steps. First, we calculated calibrated solar models for the two prescriptions of convection: the MLT and the CGM version of the full spectrum of turbulence. We then calculated RGB models for different masses, metallicities, and initial helium fractions. We compared models with data using the classical HR diagram and the squared radius vs. luminosity diagram. In the third step, we investigated the mass distribution on the red giant branch. We assumed that the mass distribution of the data set follows the present-day Galactic field stellar mass function. Finally we used the asteroseismically determined masses in the few cases where they are available.

Our conclusions are the following:

i) Lower envelope of the RGB When the mixing length theory is used with the solar convective length scale $\left(\Lambda=\alpha_{\mathrm{mlt}} H_{\mathrm{p}}\right.$ and $\left.\alpha_{\text {mlt }}=1.98\right)$, the model with $0.95 M_{\odot} \&[\mathrm{Fe} / \mathrm{H}]=-0.17$ ascends the RGB at $\approx 11.5 \mathrm{Gyr}$ i.e. the estimated age of the local Galaxy disk. This model poorly fits the lower envelope of the RGB in effective temperature or radius. The situation is not improved by lowering of the mass or the helium fraction unless we consider models older than the Universe or having an helium fraction below the Big Bang nucleosynthesis value. A solar metallicity model of $0.95 M_{\odot}$ provides a closer fit but at the expense of its age (13.7 Gyr) being hardly compatible with current constraints on the age of the Galaxy. Models with smaller convective length scales $\left(\alpha_{\text {mlt }}=1.68\right)$ reproduce the observations more closely in terms of both in effective temperatures and radii. Those models suggest two age and mass components for the lower envelope of the local RGB. The $0.95 M_{\odot} \&[\mathrm{Fe} / \mathrm{H}]=-0.17$ model reaches $10^{3} L_{\odot}$ at $11.5 \mathrm{Gyr}$ and the $1.13 M_{\odot} \&[\mathrm{Fe} / \mathrm{H}]=0$ model reaches this luminosity at $7.5 \mathrm{Gyr}$. This result interestingly agrees with Liu \& Chaboyer (2000) who estimate that the age of field stars with $[\mathrm{Fe} / \mathrm{H}]=-0.18$ is $11.7 \pm 1.9 \mathrm{Gyr}$ and the age of field stars with $[\mathrm{Fe} / \mathrm{H}]=0$ is $7.5 \pm 1.7 \mathrm{Gyr}$ (see Table 5 of these authors).

When the full spectrum of turbulence model is used with the solar convective length scale $\left(\Lambda=\alpha_{\text {cgm }} H_{\mathrm{p}}\right.$ and $\left.\alpha_{\text {cgm }}=0.77\right)$, the $0.95 M_{\odot} \&[\mathrm{Fe} / \mathrm{H}]=-0.17$ model is also too warm with respect to the lower envelope of the red giant branch. It correspondingly infers smaller radii than the observed. This is expected because when the convection is efficient (e.g. in giant star envelopes), the CGM model convective flux is roughly ten times higher than the MLT one (Canuto et al. 1996). This eventually produces higher effective temperatures. One needs a convectively less efficient model $\alpha_{\text {cgm }}=0.62$, with $0.95 M_{\odot} \&[\mathrm{Fe} / \mathrm{H}]=-0.17$ to provide a good fit regardless of whether the surface temperature or the radius is used. As in the MLT case, the CGM models suggest two ages for the cool edge of the local RGB, the low metallicity component $([\mathrm{Fe} / \mathrm{H}]=-0.17)$ being $11.8 \mathrm{Gyr}$ and the high metallicity one $([\mathrm{Fe} / \mathrm{H}]=0) 6.9$ Gyr. We did not test the more appropriate version of the full spectrum of turbulence that assumes the convective length scale $\Lambda$ to be equal to the distance $z$ to the convective boundary.

ii) Global RGB and seismic targets

We studied the mass repartition on the local RGB using 1.5 and $2.5 M_{\odot}$ models. We found that models with solar-calibrated convective lengths predict a mass distribution on the RGB that seems incompatible with the local stellar mass distribution function. The lower $\alpha_{\mathrm{mlt}}=1.68$ and $\alpha_{\text {cgm }}=0.62$ are in better but not complete agreement with the expected mass distribution. Stars with seismic mass determination offer a final way of probing surface convection efficiency. Only two stars of the sample could be used in this way. The models dedicated to them are in excellent agreement with the constraints on luminosities and radii. Confirming the previous results, they support a slightly lower than solar surface convection efficiency on the RGB.

To describe the RGB, the convective length scale needs to be slightly smaller than for the Sun in the versions of the MLT and the CGM we used. This result is suggested independently by effective temperatures, radii measurements, and the few constraints provided by asteroseismology. The decline disagrees with previous analyses of the red giant branch. We interpret this point as a consequence of our systematic use of atmosphere models with low efficiency convection. In surface layers, the specific entropy increases inwards from where the the convection begins until the region where the convection becomes adiabatic. In a solar model, $35 \%$ of this increase occurs above the optical depth $\tau=20$. But on the RGB typically $15 \%$ of the increase occurs above $\tau=20$ (as estimated from model cgm4 at $100 L_{\odot}$ ). Going from solar to RGB surface conditions means that the superadiabatic convection - and therefore the radius of the star depends less and less on the atmosphere.

Acknowledgements. L. Piau is indebted to the anonymous referee whose remarks really helped improving the quality the work. He also thanks F. Kupka and R. Samadi for their help in implementing the CGM grid of atmosphere models to CESAM. L. Piau is member of the UMR7158 of the CNRS. This work was supported by the French Centre National de la Recherche Scientifique, CNRS and the Centre National d'Études Spatiales, CNES. S. Dib aknowledges partial support from the MAGNET project of the ANR. This work received the support of PHASE, the high angular resolution partnership between ONERA, Observatoire de Paris, CNRS and University Denis Diderot Paris 7. This research took advantage of the SIMBAD and VIZIER databases at the CDS, Strasbourg (France), and NASA's Astrophysics Data System Bibliographic Services.

\section{Appendix A: New CESAM integration variables}

We note an important change in the integration scheme of the stellar evolution code that we use. The hydrostatic equilibrium equation $\left(\frac{\mathrm{d} p}{\mathrm{~d} m}=-\frac{G m \rho}{r^{2}}\right)$ and the radius-mass equation $\left(\frac{\mathrm{d} r}{d m}=\frac{1}{4 \pi \rho r^{2}}\right)$ have singularities at the center of a star. For this reason and also because the CESAM code uses piecewise polynomials to describe the stellar structure (Morel 1997), the integration variables chosen initially are $m^{2 / 3}, \xi=r^{2}$, and $\lambda=\ell^{2 / 3}$ where $m$, $r$, and $\ell$ are, respectively, the local Lagrangian mass coordinate, radius, and luminosity. This choice avoids the singularities and makes the calculations stable. However it prevents the calculation of the stellar structure in cases where the luminosity becomes negative. This situation arises in the helium cores of low mass stars when they ascend the red giant branch. The core is then devoid of any energy source (but its own slow contraction) but efficiently looses energy because of the neutrinos. At some point, the hottest region in the star becomes a spherical shell that generates an inward radiative energy flux below, i.e., a negative luminosity. We changed the integration variables of the CESAM code to $m^{1 / 4}, \xi=r^{2}$ and $\lambda=\ell$. This choice leaves no singularities, is stable, and allows the calculation of the structure even though the luminosity becomes negative. To our knowledge, this choice of integration variables has never been made with the CESAM code in previous calculations. 


\section{Appendix B: The current FST convection treatment}

There are several versions of the MLT as well as the FST model. Whenever these treatments of convection are used it is important to describe them and provide their parameters to make precise comparisons with other works possible. The current MLT convection treatment is described in detail in Piau et al. (2005), whereas the current FST version relies on the Canuto et al. (1996) equations. The same equations are used for both the atmosphere and the interior convection. The atmosphere models were computed with the ATLAS12 code (Castelli 2005) and especially for the present-day solar surface composition i.e. $X=0.7392, Z=0.0122$, and the metal repartition advocated by Asplund et al. (2005). The convective flux $F_{\text {conv }}$ is given by

$F_{\text {conv }}=K_{\text {rad }} T H_{\mathrm{p}}^{-1}\left(\nabla-\nabla_{\mathrm{ad}}\right) \Phi(S)$,

where $K_{\mathrm{rad}}=\frac{4 a c T^{3}}{3 \kappa \rho}$ is the radiative conductivity, $\mathrm{S}$ is the convective efficiency, $S=\operatorname{Ra} \times \operatorname{Pr} \propto \Lambda^{4}$, Ra and $\operatorname{Pr}$ are, respectively, the Rayleigh and Prandtl numbers of the convective flow, and $\Lambda$ is its characteristic length scale. All other symbols keep their traditional meanings.

The $\Phi(S)$ function is the ratio of convective to radiative conductivity and is given by

$\Phi(S)=F_{1}(S) F_{2}(\mathrm{~S})$,

where

$F_{1}(S)=\left(K_{o} / 1.5\right)^{3} a S^{k}\left((1+b S)^{m}-1\right)^{n}$

and

$F_{2}(S)=1+\frac{c S^{p}}{1+d S^{q}}+\frac{e S^{r}}{1+f S^{t}}$

with $K_{o}=1.7$ being the Kolmogorov constant, $a=10.8654$, $b=0.00489073, k=0.149888, m=0.189238, n=1.85011$, $c=0.0108071, p=0.72, d=0.00301208, q=0.92, e=$ $0.000334441, r=1.2, f=0.000125$, and $t=1.5$.

The equations used here are similar to those of Heiter et al. (2002). However, unlike those authors we do not adopt $\Lambda=z$, where $z$ is the distance to the boundary between radiatively stable and unstable regions. It would require substantial changes to our code to solve the stellar structure equations with $\Lambda=z$. This is because this distance $z$ is not known before the equations have been solved, and makes the problem non local. In contrast $\Lambda=\alpha_{\text {cgm }} H_{\mathrm{p}}$ is a local quantity that leads to a simpler integration scheme.

\section{References}

Alonso, A., Salaris, M., Arribas, S., Martínez-Roger, C., \& Asensio Ramos, A. 2000, A\&A, 355, 1060

Anders, E., \& Grevesse, N. 1989, Geochim. Cosmochim. Acta, 53, 197 Angulo, C., Arnould, M., Rayet, M., et al., Nucl. Phys. A, 6563

Asplund, M., Grevesse, N., \& Sauval, J. 2005, ASP Conf. Ser., Vol XXX Barban, C., Matthews, J. M., de Ridder, J., et al. 2007, A\&A, 468, 1033 Basu, S., \& Antia, H. M. 1994, JApA, 15, 143

Basu, S., \& Antia, H. M. 1995, MNRAS, 276, 1402

Basu, S., Chaplin, W. J., \& Elsworth, Y. 2010, ApJ, 710, 1596

Bernkopf, J. 1998, A\&A, 332, 127

Blackwell, D. E., Smith, G., \& Lynas-Gray, A. E. 1995, A\&A, 303, 575

Böhm-Vitense, E. 1958, Zs. f. Ap., 46, 108
Brun, A. S., Turck-Chièze, S., \& Zahn, J. P. 1999, ApJ, 525, 1032

Canuto, V. M., \& Mazzitelli, I. 1991, ApJ, 370, 295

Canuto, V. M., Goldman, I., \& Mazzitelli, I. 1996, ApJ, 473, 550

Cassisi, S., Potekhin, A. Y., Pietrinferni, A., Catelan, M., \& Salaris, M. 2007, ApJ, 661, 1094

Castelli, F. 2005, Mem. S.A. It. Suppl., 8, 25

Catelan, M. 2000, ApJ, 531, 826

Cayrel de Strobel, R., Soubiran, C., \& Ralite, N. 2001, A\&A, 373, 159

Charbonnel, C., \& Talon, S. 2005, Science, 309, 2189

Christensen-Dalsgaard, J., \& Berthomieu, G. 1991, Solar Interior and atmosphere (University of Arizona Press), 401

Claret, A. 2007, A\&A, 475, 1019

Claret, A., Diaz-Cordoves, J., \& Gimenez, A. 1995, A\&AS, 114, 247

Coc, A., Vangioni-Flam, E., Descouvemont, P., Adahchour, A., \& Angulo, C. 2004, ApJ, 600, 544

D’Antona, F., \& Mazzitelli, I. 1996, ApJ, 470, 1093

De Ridder, J., Barban, C., Carrier, F., et al. 2006, A\&A, 448, 689

De Ridder, J., Barban, C., Baudin, F., et al. 2009, Nature, 459, 398

Dib, S., Shadmehri, M., Padoan, P., Maheswar, G., Ojha, D., \& Khajenabi, F. 2010, MNRAS, 405, 401

ESA 1997, The Hipparcos and Tycho Catalogues, ESA SP, 1200

Fernandes, J., Lebreton, Y., Baglin, A., \& Morel, P. 1998, A\&A, 338, 455

Ferguson, J. W., Alexander, D. R., Allard, F., et al. 2005, ApJ, 623, 585

Ferraro, F. R., Valenti, E., Straniero, O., \& Origlia, L. 2006, ApJ, 642, 225

Frandsen, S., Carrier, F., Aerts, C., et al. 2002, A\&A, 394, L5

Gardiner, R. B., Kupka, F., \& Smalley, B. 1999, A\&A, 347, 876

Grevesse, N., \& Sauval, A. J. 1998, SSRv, 85, 161

Gustafsson, B., Edvardsson, B., Eriksson, K., et al. 2003, ASPC, 288, 331

Heiter, U., Kupka, F., van't Veer-Menneret, C., et al. 2002, A\&A, 392, 619

Holweger, H., \& Mueller, E. A. 1974, SoPh, 39, 19

Houdashelt, M. L., Bell, R. A., \& Sweigart, A. V. 2000, AJ, 119, 1448

Hutter, D. J., Johnston, K. J., Mozurkewich, D., et al. 1989, ApJ, 340, 1103

Itoh, N., Mitake, S., Iyetomi, H., \& Ichimaru, S. 1983, ApJ, 273, 774

Itoh, N., Hayashi, H., Nishikawa, A., \& Kohyama, Y. 1996, ApJS, 102, 411.

Kervella, P., Bersier, D., Mourard, D., et al. 2004a, A\&A, 428, 587

Kervella, P., Thévenin, F., Di Folco, E., \& Ségransan, D. 2004b, A\&A, 426, 297

Komatsu, E., Dunkley, J., Nolta, M. R., et al. 2009, ApJS, 180, 330

Krauss, L. M., \& Chaboyer, B., Science, 299, 65

Krishna Swamy, K. S. 1966, ApJ, 145, 174

Kroupa, P. 2002, Science, 295, 82

Kroupa, P. 2007, ASP Conf. Ser., in press [arXiv: astro-Ph/0703124]

Kupka, F. 1996, ASP Conf. Proc., 108, 73

Kurucz, R. L. 1993, CD-ROM 13, Cambridge, SAO

Liu, W. M., \& Chaboyer, B. 2000, ApJ, 544, 818

Ludwig, H. G., Freytag, B., \& Steffen, M. 1999, A\&A, 346, 111

Mallik, S. V. 1998, A\&A, 338, 623

Mathis, S., Talon, S., Pantillon, F.-P., \& Zahn, J.-P. 2008, Sol. Phys., 251, 101

Mazumdar, A., Mérand, A., Demarque, P., et al. 2009, A\&A, 503, 521

Mazzitelli, I. 1999, ASPC, 173, 77

Mazzitelli, I., D’Antona, F., \& Caloi, V. 1995, A\&A, 302, 382

McWilliam, A. 1990, ApJ Suppl. Ser., 74, 1075

Mérand, A., Kervella, P., Barban, C., et al. 2010, A\&A, 517, A64

Montalban, J., Kupka, F., D’Antona, F., \& Schmidt, W. 2001, A\&A, 370, 982

Montalban, J., D’Antona, F., Kupka, F., \& Heiter, U. 2004, A\&A, 416, 1081

Monteiro, M. J. P. F. G., Christensen-Dalsgaard, J., \& Thompson, M. J. 1996, A\&A, 307, 624

Morel, P. 1997, A\&AS, 124, 597

Morel, P., van't Veer, C., Provost, J., et al. 1994, A\&A, 286, 91

Nordlund, ke, Stein, R. F., \& Asplund, M. 2009, LRSP, 6, 2

Piau, L., Ballot, J., \& Turck-Chièze, S. 2005, A\&A, 430, 571

Proffitt, C. R., \& Michaud, G. 1993, ASP Conf. Ser., 40, 246

Richichi, A., Percheron, I., \& Khristoforova, M. 2005, A\&A, 431, 773

Salaris, M., Cassisi, S., \& Weiss, A. 2002, PASP, 114, 375

Samadi, R., Kupka, F., Goupil, M. J., Lebreton, Y., \& van't Veer-Menneret, C. 2006, A\&A, 445, 233

Schaller, G., Schaerer, D., Meynet, G., \& Maeder, A. 1992, A\&AS, 96, 269

Stothers, R. B., \& Chin, C. W. 1997, ApJ, 478, L103

Thévenin, F., \& Idiart, T. P. 1999, ApJ, 521, 753

Thévenin, F., Kervella, P., Pichon, B., et al. 2005, A\&A, 436, 262

Turck-Chièze, S., Couvidat, S., Piau, L., et al. 2004, PhRvwL, 93, 1102

VandenBerg, D. A., Edvardsson, B., Eriksson, K., \& Gustafsson, B. 2008, ApJ, 675,763

Wittkowski, M., Hummel, C. A., Aufdenberg, J. P., \& Roccatagliata, V. 2006, A\&A, 460, 843 